\title{
Effects of Magnetic Fields on Bar Substructures in Barred Galaxies
}

\section{Woong-Tae Kim}

Center for the Exploration of the Origin of the Universe (CEOU), Department of Physics \& Astronomy, Seoul National University, Seoul 151-742, Republic of Korea

email: wkim@astro.snu.ac.kr

\begin{abstract}
To study the effects of magnetic fields on the properties of bar substructures, we run two-dimensional, ideal MHD simulations of barred galaxies under the influence of a nonaxisymmetric bar potential. In the bar regions, magnetic fields reduce density compression in the dust-lane shocks, while removing angular momentum further from the gas at the shocks. This evidently results in a smaller and more distributed ring, and a larger mass inflows rate to the galaxy center in models with stronger magnetic fields. In the outer regions, an MHD dynamo due to the combined action of the bar potential and background shear operates, amplifying magnetic fields near the corotation resonance. In the absence of spiral arms, the amplified fields naturally shape into trailing magnetic arms with strong fields and low density. The reader is refereed to Kim \& Stone (2012) for a detailed presentation of the simulation outcomes.
\end{abstract}

Keywords. magnetohydrodynamics, galaxies: ISM, galaxies: spiral, shock waves
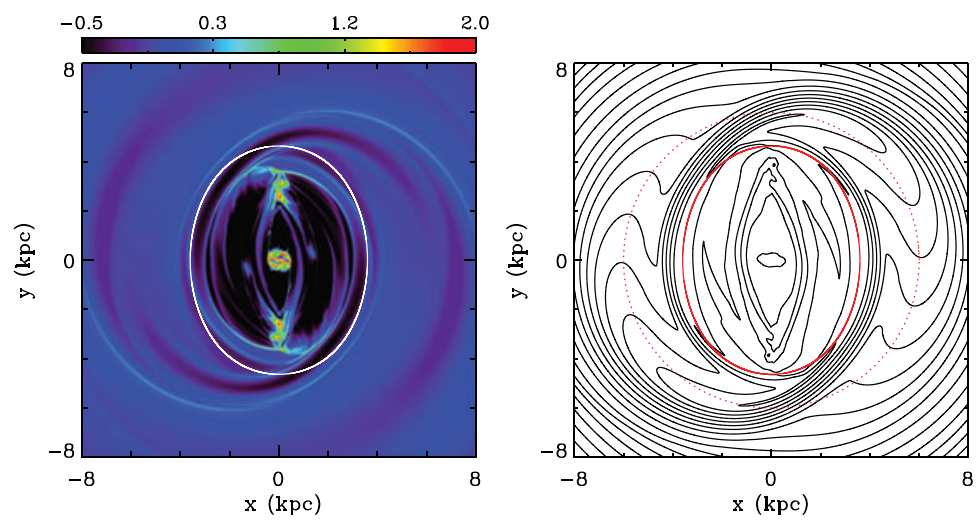

Figure 1. Distribution of density in logarithmic scale (left) and magnetic fields (right) at $t=0.3 \mathrm{Gyr}$ in the $\beta=3$ model, where $\beta$ is the plasma parameter. The solid oval represents the outermost $x_{1}$-orbit, relative to which gas responses are different dramatically. The dotted line in the left panel draws the corotation radius, around which magnetic fields are amplified.

\section{Acknowledgements}

This work was supported by the National Research Foundation of Korea (NRF) grant funded by the Korean government (MEST), No. 2010-0000712.

\section{Reference}

Kim, W.-T. \& Stone, J. M. 2012, ApJ, 751, 124 